Accepted refereed manuscript of: Goutcher R \& Wilcox LM (2021) Surface slant impairs disparity discontinuity discrimination. Vision

Research, 180, pp. 37-50.https://doi.org/10.1016/j.visres.2020.12.001

(c) 2020, Elsevier. Licensed under the Creative Commons Attribution-NonCommercial-NoDerivatives 4.0 International http://

creativecommons.org/licenses/by-nc-nd/4.0/

\title{
Surface Slant Impairs Disparity Discontinuity Discrimination
}

\author{
Ross Goutcher ${ }^{1} \&$ Laurie M. Wilcox ${ }^{2}$ \\ Corresponding author email: ross.goutcher@stir.ac.uk \\ ${ }^{1}$ Psychology, Faculty of Natural Sciences \\ University of Stirling, Stirling, UK \\ FK9 4LA \\ ${ }^{2}$ Department of Psychology, Centre for Vision Research \\ York University, Toronto, ON, Canada \\ Draft Publication. Please do not cite without prior permission
}

\begin{abstract}
November 2020
Abstract: Binocular disparity signals are highly informative about the three-dimensional structure of visual scenes, including aiding the detection of depth discontinuities between surfaces. Here, we examine factors affecting sensitivity to such surface discontinuities. Participants were presented with random dot stereograms depicting two planar surfaces slanted in opposite directions and were asked to judge the sign of the depth discontinuity created where those surfaces met. Although the judgement was focussed on the adjacent edges, the precision of depth discontinuity discrimination depended upon the slant of the two surfaces: increasing surface slants to $\pm 60^{\circ}$ increased discontinuity discrimination thresholds by, on average, a factor of 5 . Control experiments examining discontinuity discrimination across surfaces with identical slants showed either biases in discontinuity judgements or reduced threshold elevation. These results suggest that sensitivity to depth discontinuities is affected by processing limitations in both local absolute disparity measurement mechanisms and mechanisms selective for disparity differences. As further evidence in support of this conclusion, we show that our results are well-described by a model of discontinuity discrimination based on the encoding of local differences in relative disparity.
\end{abstract}

Keywords: Binocular vision; stereoacuity; absolute disparity; relative disparity 


\section{Introduction}

Binocular disparities, the small positional differences arising due to viewing the world with two frontfacing eyes, provide the human visual system with high precision information about the threedimensional (3D) structure of the world. Judgements of relative depth for isolated points can be successfully made for disparities of as little as 2 seconds of arc (Howard, 1919), with an estimated $80 \%$ of adults in western populations having stereoacuities of 30 seconds of arc or better (Coutant \& Westheimer, 1993). Critically, however, these limits on stereoacuity depend upon exact stimulus configurations. For example, the addition of a pedestal disparity, where depth differences are presented away from the plane of fixation, is known to severely impair the ability to judge relative depth (e.g. Badcock \& Schor, 1985; Blakemore, 1970; Westheimer \& McKee, 1978).

While measurements of stereoacuity have been reported for numerous stimulus configurations, one important observation is that depth judgements depend upon the structure of stereoscopic surfaces. Several researchers have reported that intervening stereoscopic structures can impair the ability to judge relative depth. For simple line stimuli, the presence of horizontal lines joining pairs of vertical lines raises thresholds for the disparity required to judge which line is closer in depth (Mamassian \& Zannoli, 2020; McKee, 1983; Mitchison \& Westheimer, 1984; Westheimer, 1979).

Recently, a number of other authors (Cammack \& Harris, 2016; Deas \& Wilcox, 2014, 2015; Goutcher, Connolly \& Hibbard, 2018) have shown that intervening disparity information can also impair the ability to discriminate between suprathreshold depth differences: stimuli containing disparities that vary continuously over space (e.g. slanted surfaces) appear to have less depth than stimuli containing discontinuous step changes in disparity. A number of factors have been implicated in these effects. Deas and Wilcox $(2014,2015)$ have shown that Gestalt grouping principles play a role in reducing the perception of quantitative depth. Meanwhile, Goutcher et al (2018) have shown that perceived depth depends upon both surface continuity and the structure of intervening discontinuities. Disparity-defined depth discontinuities can increase or decrease perceived depth, depending on whether the sign of the discontinuity is the same as, or in opposition to, the change in disparity across the surface. These authors suggested that such effects may be driven by the properties of mechanisms encoding disparity differences across space.

While these studies have highlighted the effects of intervening surface structures on judgements of stereoacuity and on the magnitude of perceived depth, they do not tell us whether such effects are also present for other surface structures. In particular, it is important to establish whether stereoacuity judgements are affected by the structure of adjacent surfaces. Any impairments arising due to adjacent surfaces could limit the ability to detect disparity-defined object boundaries or judge their relative depth, with subsequent consequences for the use of disparity for figure-ground segmentation or the breaking of camouflage. In this paper, we examine the effects of adjacent surface structures on stereoacuity judgements at disparity-defined discontinuities and consider the neural processes that may underpin any such effects.

Within the brain, neurophysiological and brain imaging evidence from both humans and non-human animals has pointed to a complex network of systems, across multiple areas of cortex, supporting the measurement and use of binocular disparity signals (cf. Neri, 2005; Parker, 2007; Verhoef, Vogels \& Janssen, 2016). Within this network, cortical processing of binocular disparity changes from early selectivity for absolute (i.e. retinal) disparities to selectivity for disparity differences (i.e. relative disparities). Observed biases and limitations in stereoacuity judgements may therefore depend upon activity at one or more of these processing stages. In this paper, we consider three possible sets of mechanisms that may limit performance in stereoacuity tasks: the initial measurement of absolute disparities; the measurement of relative disparities, and the application of grouping processes to the perception of disparity-defined form.

At the level of initial disparity measurement, selectivity for absolute disparities has been found in V1 of the macaque and in area 17 of the cat (Cumming \& Parker, 1999; DeAngelis, Ohzawa \& Freeman, 1991; Ohzawa, DeAngelis \& Freeman, 1990). The operation of these mechanisms has been wellaccounted for by the disparity-energy model (e.g. Fleet, Wagner \& Heeger, 1996; Read, 2005), which can be considered as a process of cross-correlation (Banks, Gepshtein \& Landy, 2004; Read, 2005). 
Variations on such cross-correlation-based models have been used to account for a number of performance impairments in stereoscopic vision, including limitations in disparity frequency sensitivity (Banks et al, 2004), disparity gradient limits (Filippini \& Banks, 2009), upper disparity limits for the detection of cyclopean gratings (Allenmark \& Read, 2010, 2011) and coarse scale preferences in similarity-based correspondence matching (Goutcher \& Hibbard, 2014). The extent to which such models account for human performance is often dependent on spatial integration processes. Image areas viewed by larger windows for cross-correlation (i.e. larger areas for the spatial pooling of binocular simple cell responses) are more likely to contain spatial variations in disparity. Such variations limit the selectivity of cross-correlation-based responses, resulting in an increased uncertainty on any local estimates of absolute disparity (cf. Filippini \& Banks, 2009; Goutcher \& Hibbard, 2020).

Beyond this early selectivity for absolute disparities, cells tuned to relative disparities have been found in multiple cortical areas, including both V2 (Bredfeldt \& Cumming, 2006; Thomas, Cumming \& Parker, 2002) and V4 (Fang et al, 2018; Umeda, Tanabe \& Fujita, 2007). Goutcher and colleagues (Goutcher et al, 2018; Goutcher \& Hibbard, 2020) have suggested that this stage of processing plays a critical role in producing observed biases in the perceived depth of continuous and discontinuous surfaces (Cammack \& Harris, 2016, Goutcher et al, 2018) and in the masking of disparity-defined surface structures by random disparity masking stimuli (Goutcher \& Hibbard, 2020). Mechanisms selective for relative disparity may also be involved in other stereoacuity biases, including observed effects of slanted or curved reference planes (Glennerster \& McKee, 1999, 2004; Hornsey, Hibbard \& Scarfe, 2016; Petrov \& Glennerster, 2004, 2006).

Evidence for the specific neural mechanisms underlying depth-based grouping is less prevalent. Qiu and von der Heydt (2005) argued that relative disparity selective neurons in V2 are also influenced by Gestalt grouping rules for the encoding of object boundaries, while Samonds and colleagues (Samonds, Potetz \& Lee, 2009; Samonds, Tyler \& Lee, 2016) have presented evidence of recurrent connections between disparity selective cells in V1. Such recurrent connections could, in principle, support grouping processes for rules such as proximity, good continuation and similarity via the encoding of association fields (Field, Hayes \& Hess, 1993; Field \& Hayes, 2004).

To assess the contribution of each of these processing stages, we examined whether the ability to discriminate relative depth at a disparity discontinuity was affected by the presence of slanted surfaces on either side of the discontinuity. We measured stereoacuity thresholds for such stimuli, varying the gap between surfaces to see whether any effects of adjacent surfaces were dependent on the distance between the discontinuous edges. We show that stereoacuity thresholds are impaired when surfaces on either side of the discontinuity are slanted in opposing directions but find no consistent effect of varying the gap between edges. In a series of control experiments, we show that the effects of surface slant are markedly reduced when discontinuities occur on continuously slanted surfaces and when discontinuities are orthogonal to surface slant. We further show that these results are well-described by a model based on the encoding of local differences in relative disparity. Together, these results suggest that slant effects for judgements of disparity discontinuities are primarily driven by mechanisms operating at least at the level of relative disparity measurement.

\section{Experiment 1: Surface Slant Impairs Judgements of Discontinuities in Depth}

Experiment 1 examined the effects of disparity-defined slant on depth discontinuity discrimination. Participants were presented with stimuli containing a single depth discontinuity, where the surfaces on either side of that discontinuity were slanted in opposite directions in depth. We measured thresholds for the discrimination of the sign of the discontinuity as a function of surface slant. These thresholds were measured on both vertical and horizontal slant axes, with the orientation of the depth discontinuity always matching the axis of slant. To evaluate the effect of proximity-based grouping on threshold elevation we varied the distance between the edges of the two slanted planes, i.e. we changed the size of the gap between the surfaces. If proximity between surfaces impairs the ability to discriminate the relative depth of discontinuous edges due to grouping processes, we should expect less threshold elevation as the gap between surface edges increases. 


\subsection{Methods}

\subsubsection{Participants}

There were a total of 7 observers in Experiment 1, including author RG; four completed all test conditions and three completed either horizontal or vertical discontinuity conditions resulting in a total of 6 participants for the vertical discontinuity condition and 5 for the horizontal discontinuity condition. All had normal or corrected-to-normal visual acuity and had stereoacuity thresholds of at least 1 arcmin, as tested by the RanDot2 stereo test (Vision Assessment Corp., Elk Grove Village, IL). Note, this measure of stereoacuity is indicative and was not used as a cut-off criterion for participation. All participants were experienced psychophysical observers and, apart from author RG, were naïve as to the purpose of the experiment. The experimental protocol was approved by the Stirling University Ethics Panel.

\subsubsection{Stimulus \& Apparatus}

Stimuli presentation was controlled by a Mac Pro computer, with a $49 \times 31 \mathrm{~cm}$ Apple Cinema HD display (Apple Inc., Cupertino, CA). Display resolution was $1920 \times 1200$ pixels, with a refresh rate of $60 \mathrm{~Hz}$. The display was calibrated using a SpyderPro2 calibration device (DataColor, Dietlikon, Switzerland) to ensure a linear greyscale, with luminance outputs ranging from $0.18 \mathrm{cdm}^{-2}$ to $45.7 \mathrm{cdm}^{-2}$. Viewing distance was $76.4 \mathrm{~cm}$, with each pixel subtending $1.1 \mathrm{arcmin}$. Binocular stimulus presentation made use of a modified Wheatstone mirror stereoscope, calibrated to ensure consistency of eye convergence and accommodation, with head movements restricted using a HeadSpot (UCHO, Houston, TX) chin rest. All stimuli were created and presented using Matlab (Mathworks Inc., Natick, MA.) and the Psychophysics Toolbox (Brainard, 1997; Pelli, 1997; Kleiner, Brainard \& Pelli, 2007).

Experimental stimuli were random dot stereograms (RDS) depicting pairs of planar surfaces (see example stimuli in Figure 1). Each surface was displayed within a circular area measuring $4.4^{\circ}$ in diameter, surrounded by a circular zero disparity fixation plane comprised of randomly positioned squares. The diameter of the inner edge of the fixation plane was $5.6^{\circ}$, while that of the outer edge was $6.6^{\circ}$. Fixation plane squares measured between 1.6 and 14 arcmin across, while surface stimulus dots were white anti-aliased circles of diameter $3.3 \mathrm{arcmin}$. Anti-aliased dots were defined using a scaled, inverted two-dimensional quadratic function, with the central location shifted in steps of one tenth of a pixel (cf. Georgeson, Freeman \& Scott-Samuel, 1996). The two stimulus surfaces were separated by a blank region of $6.6 \mathrm{arcmin}, 13.2 \mathrm{arcmin}$ or $26.4 \mathrm{arcmin}$. The random-dot surfaces either side of this blank region were slanted at $\pm 60^{\circ}, \pm 30^{\circ}$ or $0^{\circ}$ through the addition of horizontal binocular disparities along the horizontal or vertical axes. Surface slants were of opposite sign either side of the blank region, with the slant of the left surface used to describe the stimulus condition (top surface for horizontal slants). Thus, for slants of $-60^{\circ}$ and $-30^{\circ}$, both surfaces receded behind the plane of fixation with increasing distance from the blank central region, while for slants of $60^{\circ}$ and $30^{\circ}$ both surfaces protruded in front of the fixation plane. 

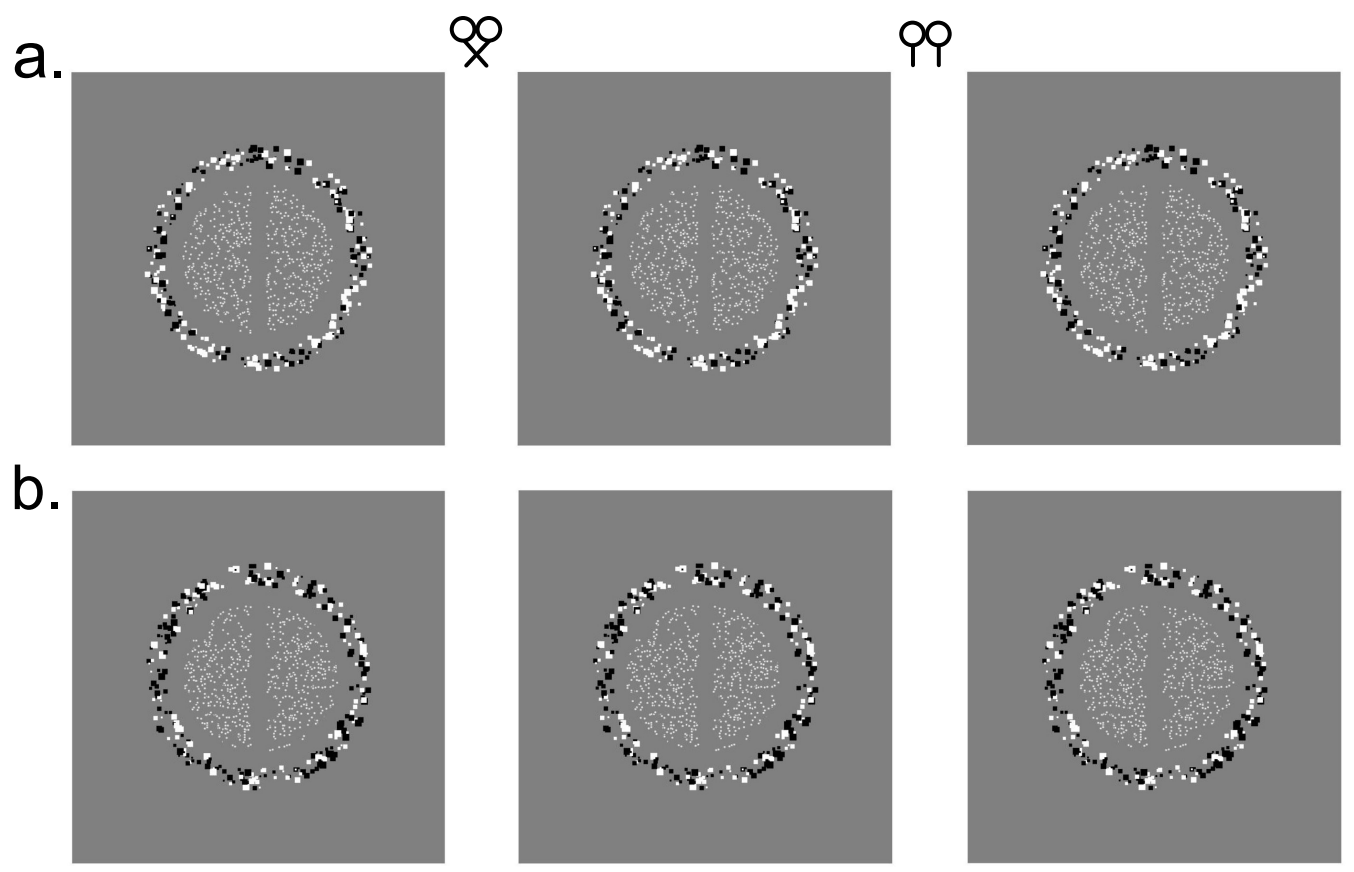

Figure 1. Example of the stimuli used in Experiment 1, arranged for crossed (left \& centre) and uncrossed (centre and right) fusion. Stimuli depict vertical depth discontinuities, with surfaces slanted around a vertical axis (a) Discontinuous step edge in depth, where neighbouring surfaces are fronto-parallel. (b) Discontinuous step edge in depth, with oppositely slanted surfaces at $\pm 60^{\circ}$.

To introduce depth discontinuities, surfaces were shifted in opposing directions in depth via the addition of horizontal binocular disparity to each stimulus element. These disparities shifted each surface in its entirety forwards or backwards in depth, allowing surface slant to be maintained despite the introduction of the depth discontinuity. For the purposes of data analysis, negative discontinuity values were used to indicate stimuli where the edge on the left of the blank region (or above the blank region for horizontal slants) was closer. Positive values indicated that the edge to the right of the blank region was closer (or below, for horizontal slants).

\subsubsection{Design \& Procedure}

On each experimental trial, participants were presented with a RDS in a single-interval, twoalternative-forced-choice design. As described above, each trial contained two planar surfaces, separated by a small gap, with a depth discontinuity between neighbouring surface edges. The participants' task was to determine whether the edge of the left or right surface was closer in depth, responding via a key press. The sign and magnitude of the depth discontinuity was varied over 9 levels using the method of constant stimuli, in order to measure thresholds for discontinuity discrimination at each of 5 surface slant arrangements. Discontinuity step sizes were determined individually in an initial block of trials which also served as practice. The test range varied across participants and surface slant, with maximum disparities of 2.5 arcmin for surface slants of $\pm 60^{\circ}, 2$ arcmin for $\pm 30^{\circ}$ slants and 1.4 arcmin for surface slants of $0^{\circ}$. Stimuli were presented for $300 \mathrm{~ms}$, preceded by the $500 \mathrm{~ms}$ presentation of a fixation cross. Participants viewed each stimulus condition 20 times in random order, over a series of blocks.

While multiple levels of surface slant and discontinuity magnitude were presented within each block, both the discontinuity orientation and the gap between discontinuities were kept constant within blocks. Only 3 participants collected data at the 26.4 arcmin gap size for vertical discontinuities. Similarly, data for horizontal discontinuities were collected for gaps of size 6.6 arcmin and 13.2 arcmin only, with data for two participants collected at the 6.6 arcmin gap size only.

\subsection{Results \& Discussion}

To examine the effects of surface slant manipulations on discontinuity discrimination, we fit cumulative Gaussian distributions to the proportion of "right edge closer" responses as a function of 
disparity. Examples of these functions are shown for a vertical discontinuity with a gap size of 6.6 arcmin for one participant in Figure 2a. The slope of the cumulative Gaussian function, given by the standard deviation $\sigma$, was taken as a measure of the precision of discontinuity discrimination, equivalent to the measurement of a just noticeable difference (JND) taken at the 0.84 point on the cumulative Gaussian.

To assess the effects of surface slant on the precision of discontinuity discrimination, JND measurements were normalised for each participant by dividing by their JND for fronto-parallel surfaces. This normalisation allows for the comparison of slant-related threshold elevations, regardless of individual differences in general stereoacuity. Mean JNDs for fronto-parallel surfaces prior to normalisation were 9.5 arcsec, 10.7 arcsec and 9.8 arcsec, for 6.6 arcmin, 13.2 arcmin and 26.4 arcmin gaps, respectively, with maximum and minimum JND values of 16.8 and 5.2 arcsec across participants and gap sizes. Figure $2 \mathrm{~b}$ plots normalised JND ratios as a function of surface slant for each gap size and each participant, for vertical discontinuities only. These figures clearly show an increase in JND, and thus a decrease in the precision of discontinuity discrimination, with increasing surface slant. JNDs increased by, on average, a factor of 5.38 (with $95 \%$ confidence intervals of \pm 0.78 ) for surface slants of $\pm 60^{\circ}$ with a gap size of 6.6 arcmin. This decreased slightly for larger gap sizes, to a factor of $3.75( \pm 1.09)$ for a 13.2 arcmin gap and a factor of $4.05( \pm 1.51)$ for a 26.4 arcmin gap.
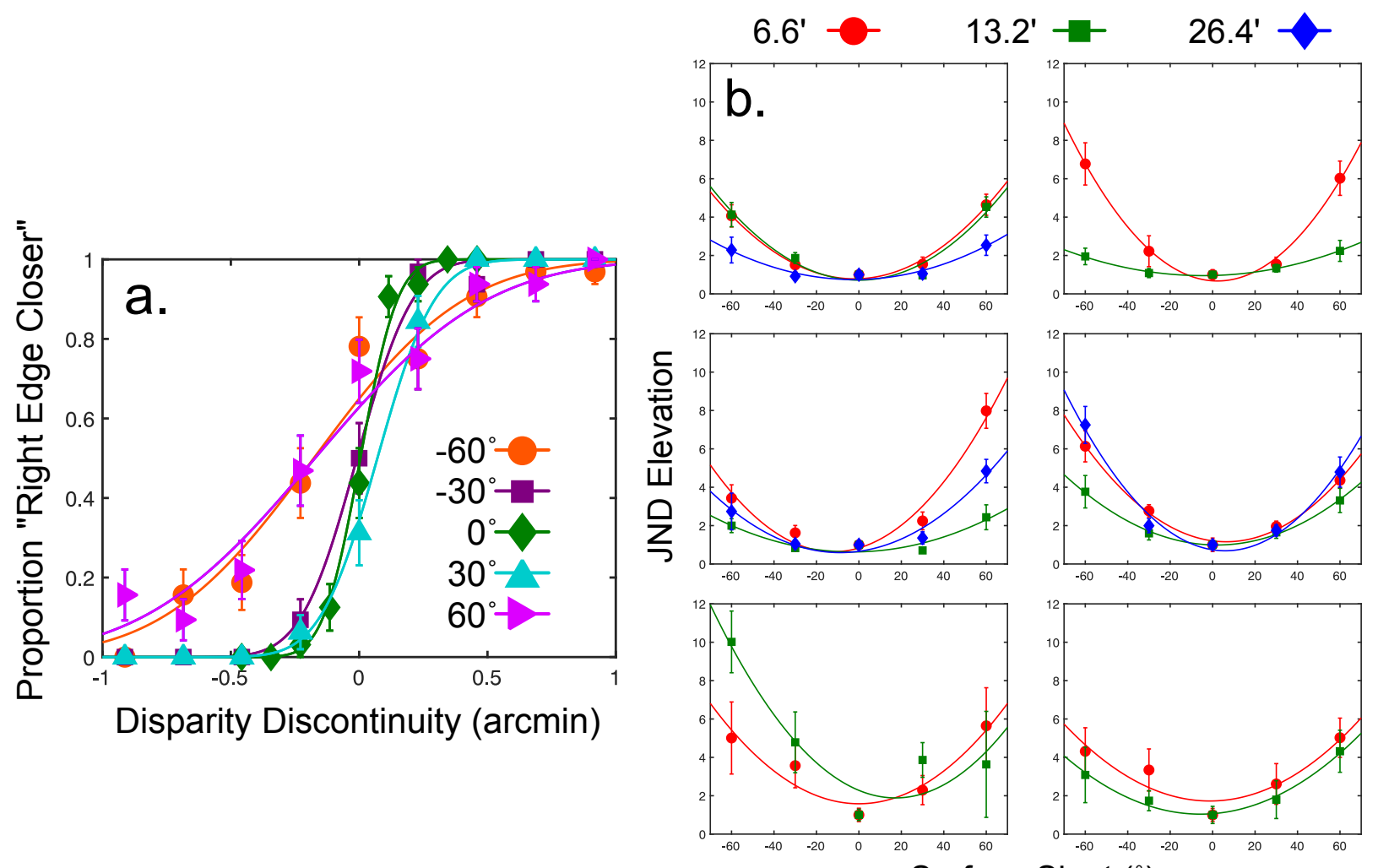

Surface Slant $\left(^{\circ}\right)$

Figure 2. Results of Experiment 1, showing the impact of slant about a vertical axis on discontinuity discrimination. (a) Psychometric functions at each slant angle, for a single participant at a gap size of 6.6 arcmin. Error bars show binomial standard errors (b) Normalised JND ratios as a function of surface slant, for each participant and each gap size. Error bars show standard deviation of the distribution of bootstrapped estimates of normalised JNDs.

To provide a measure of this effect of slant on discontinuity discrimination, normalised JND ratios were fitted with a quadratic function $\sigma=a x^{2}+b x+c$. This provided a good fit to the data across all conditions, with a mean adjusted $r^{2}$ value of 0.897 (with maximum and minimum values of 0.99 and 0.69 , respectively). Mean values for the quadratic coefficient ' $a$ ' were $0.0012( \pm 0.0002)$ for a gap of 6.6arcmin, $0.0007( \pm 0.0003)$ for a gap of 13.2arcmin and $0.0009( \pm 0.0006)$ for a gap of 26.4arcmin. The value of these coefficients was significantly greater than zero on a series of Holm-Bonferroni 
corrected one-tailed, one-sample t-tests: $t_{5}=11.416, p=0.00005$ for the 6.6 arcmin gap, $t_{5}=4.824$, $p=0.0024$ for the 13.2 arcmin gap and $t_{2}=2.935, p=0.045$ for the 26.4 arcmin gap.

Results were very similar for slants about a horizontal axis (see Figure 3 ), where cumulative Gaussian distributions were fit to the proportion of "bottom edge closer" responses for each surface slant. In these conditions, slopes increased, at a slant of $\pm 60^{\circ}$, by a factor of $4.49( \pm 0.96)$ for the 6.6 arcmin gap and by a factor of $3.62( \pm 1.44)$ for the 13.2 arcmin gap. Quadratic coefficient fits were again significantly greater than zero, with means of $0.001( \pm 0.0003)$ for the 6.6 arcmin gap and $0.0007( \pm 0.0003)$ for the 13.2 arcmin gap $\left(t_{4}=6.149, p=0.0018\right.$ and $t_{2}=4.158, p=0.0266$, respectively on Holm-Bonferroni corrected one-sample t-tests). The quadratic function again provided a good fit to the data, with adjusted $r^{2}$ values ranging between 0.876 and 0.967 , with a mean value of 0.934 .
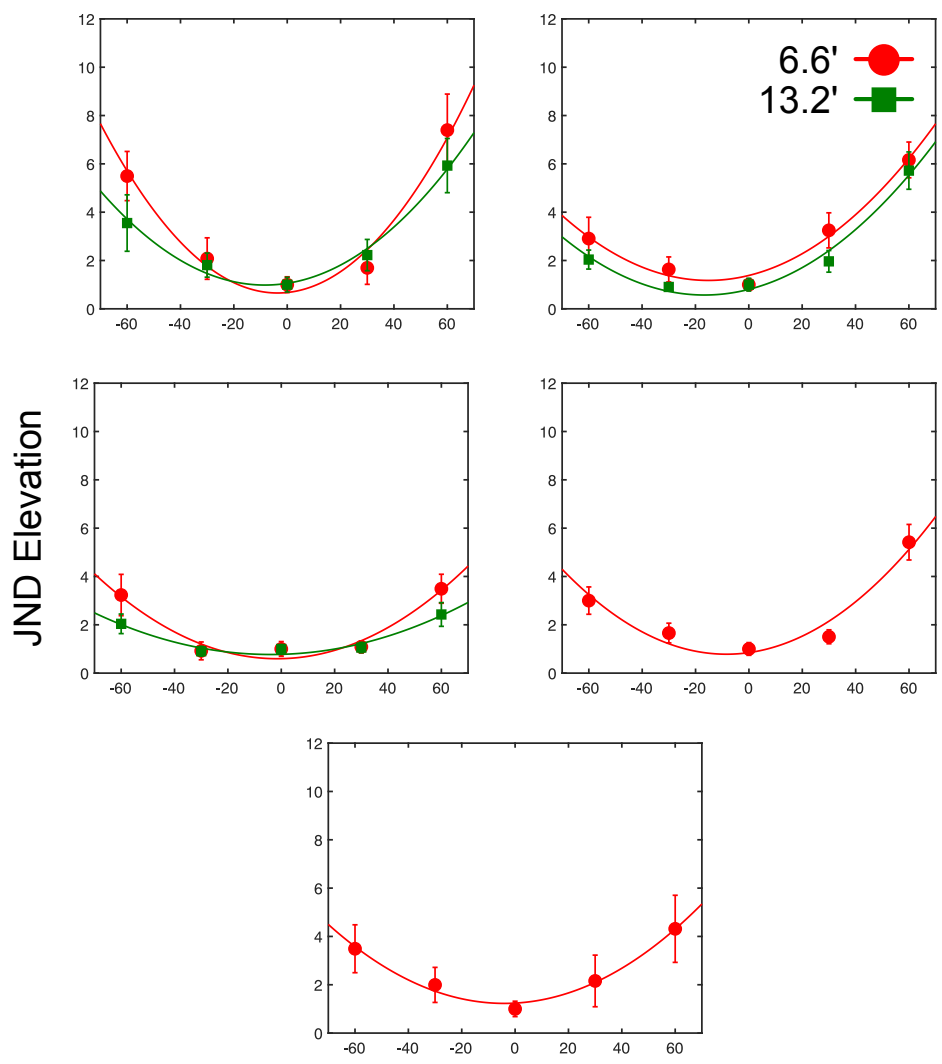

Surface Slant $\left({ }^{\circ}\right)$

Figure 3. Results of Experiment 1 for surfaces slanted about a horizontal axis. Plots show normalised JND ratios as a function of slant for each participant and gap size. Error bars show the standard deviation of the distribution of bootstrapped estimates of normalised JNDs.

While there are clear effects of adjacent surface slant on discontinuity discrimination judgements, the manipulation of the gap size between surface edges failed to produce any clear pattern of results. No significant differences were found between quadratic coefficients for vertical discontinuities: $t_{5}=$ 1.7264, $p=0.1449 ; t_{2}=0.8405, p=0.4891 ; t_{2}=0.5482, p=0.6386$ for comparisons between 6.6 and 13.2 arcmin gaps, 6.6 and 26.4 arcmin gaps and between 13.2 and 26.4 arcmin gaps, respectively. Similarly, there was no significant difference between 6.6 and 13.2 arcmin gaps for horizontal discontinuities $\left(t_{2}=2.1587, p=0.1635\right)$. We present comparisons with the 26.4 arcmin gap and for horizontal slants for the sake of completeness, fully aware that the small participant numbers limit the ability to draw strong conclusions from this negative result.

The effects of surface slant shown here complement previously reported effects of surface structure on perceived depth. Just as intervening surface structures can impair judgements of relative depth, so too can adjacent surface structures impair depth judgements between neighbouring surface edges. The mechanisms underpinning these effects remain unclear, however. Stereoacuity 
judgements could have been impaired by either increased uncertainty in the measurement of absolute disparity, or by the tuning properties of relative disparity selective mechanisms. To distinguish between these possibilities, in Experiments 2 and 3, below, we examined the effects of different surface slant arrangements on stereoacuity judgements.

\section{Experiment 2: Discontinuity Discrimination is Biased by Slant Sign}

Results from Experiment 1 showed that discontinuity discrimination judgements were impaired when surfaces either side of the discontinuity were slanted in depth. To determine the extent to which these impairments were due to the effects of surface slant on local measurements of absolute disparity, in Experiment 2 we measured discontinuity discrimination thresholds for cases where the two surfaces on either side of the discontinuity had the same slant. This is a critical manipulation: if the effect of slant on stereoacuity is simply the product of increased uncertainty in absolute disparity measurement, changing the relative sign of the surfaces should have no effect on threshold elevations. If, however, slant effects are due to the properties of mechanisms measuring relative disparity, then slanted surfaces of the same sign represent a greatly simplified problem. In such stimuli, local relative disparity is constant except at the discontinuous boundary. We return to this point in greater detail in section 5 , below.

\subsection{Methods}

\subsubsection{Participants}

Experiment 2 was completed by 5 participants, including author RG. All had previously participated in Experiment 1.

\subsubsection{Stimulus \& Apparatus}

The equipment for Experiment 2 was identical to that used in Experiment 1, as were all viewing parameters and equipment calibrations, with the exception that surfaces either side of the discontinuous boundary were of the same slant. All surfaces were slanted about a vertical axis. Surface slant was again either $\pm 60^{\circ}, \pm 30^{\circ}$ or $0^{\circ}$, with positive values indicating a left-near, right-far slant and negative values indicating the opposite. An example stimulus is shown in Figure 4.

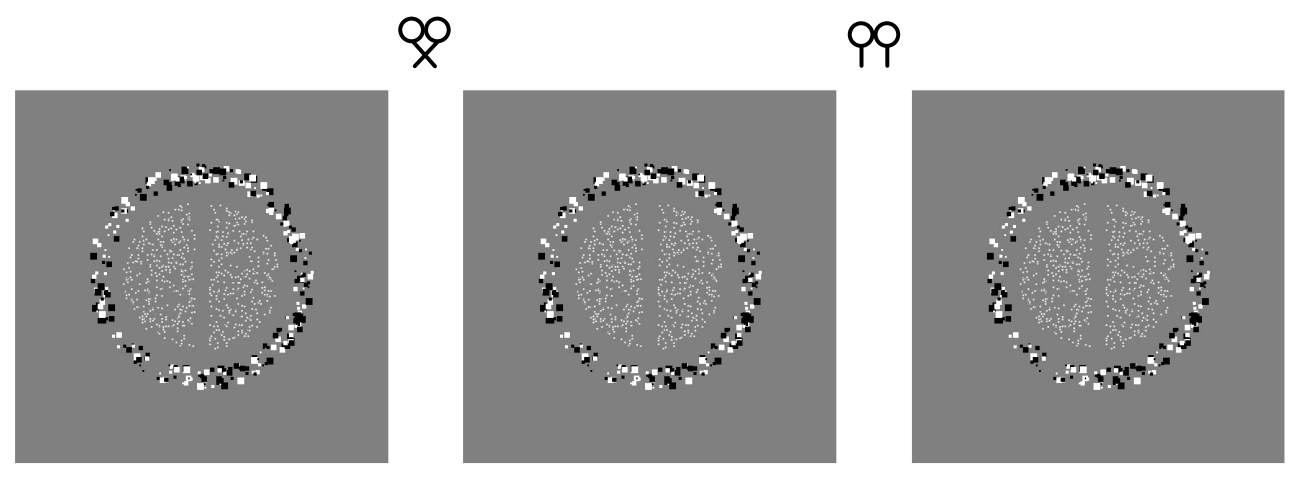

Figure 4. Example of the stimuli used in Experiment 2, arranged for crossed (left \& centre) and uncrossed (centre \& right) fusion. Stimuli depict vertical depth discontinuities where adjoining surfaces are identically slanted about a vertical axis. When correctly fused, depicted surfaces are slanted at $60^{\circ}$ left-near, right-far, with a right-near discontinuity.

\subsubsection{Design \& Procedure}

The experimental design and procedure were the same as described for Experiment 1 . Following the initial collection of pilot data, the range of discontinuity disparities were determined individually for each surface slant level. As before, participants viewed 9 levels of discontinuity magnitude at each of 5 surface slants. Final discontinuity magnitudes were again set for each participant following the completion of a block of practise trials. Maximum disparity values ranged between \pm 2.2 arcmin at slants of $\pm 60^{\circ}, \pm 1.8$ arcmin at slants of $\pm 30^{\circ}$ and \pm 1.15 arcmin at the $0^{\circ}$ slant. Unlike Experiment 1 , discontinuity levels were not centred on zero, as initial pilot data collection showed that there were 
significant biases in the judgement of relative depth (see full details in section 3.2, below). Once again, stimuli were presented for $300 \mathrm{~ms}$, preceded by the $500 \mathrm{~ms}$ presentation of a fixation cross. Participants viewed the stimuli in random order over a series of blocks, with each participant completing a minimum of 20 repeated trials of each experimental stimulus. As before, gap size varied between, but not within, blocks. All participants collected data at gap sizes of 6.6 arcmin, and 13.2 arcmin; three participants also collected data at a gap size of 26.4 arcmin.

\section{2 Results \& Discussion}

As before, proportion "right edge closer" responses were taken as a function of disparity and used to fit cumulative Gaussian distributions with the slope of the fitted functions taken as a measure of the precision of discontinuity discrimination (see Figure 5a). Estimates of slope at each surface slant were again normalised by dividing by the fitted slope value for $0^{\circ}$ slanted stimuli (averaging 6.9, 6.3 and 7.4 arcsecs for 6.6, 13.2 and 26.5 arcmin gaps, respectively, with maximum and minimum values of 11.9 and 5.9 arcsec), to provide measures of normalised JND ratios. Normalised JNDs are plotted, for each participant, in Figure $5 \mathrm{~b}$, together with fitted quadratic functions describing the effect of slant on discontinuity discrimination. From these figures, it is evident that surface slant continued to have a negative effect on discontinuity discrimination, although somewhat less so than in Experiment 1. Average normalised JND ratios were $3.372( \pm 0.691), 3.635( \pm 0.779)$ and $5.532( \pm 2.098)$ at gap sizes of 6.6, 13.2 and 26.4 arcmin, respectively. Mean values for the fitted quadratic coefficient at these gap sizes were $0.0007( \pm 0.0002), 0.0008( \pm 0.0003)$ and $0.0013( \pm 0.0009)$. With the exception of the 26.4 arcmin gap, where participant numbers were low, quadratic coefficient values were significantly greater than zero $\left(t_{4}=6.767, p=0.0012 ; t_{4}=4.725, p=0.0046\right.$ and $t_{2}=2.825, p=0.0529$ on onetailed, Holm-Bonferroni corrected, one sample t-tests, for gap sizes of 6.6, 13.2 and 26.4arcmin, respectively). Again, the data were well fit by a quadratic function, with adjusted $r^{2}$ values ranging between 0.754 and 0.997 across participants and gap sizes, with a mean value of 0.897 .
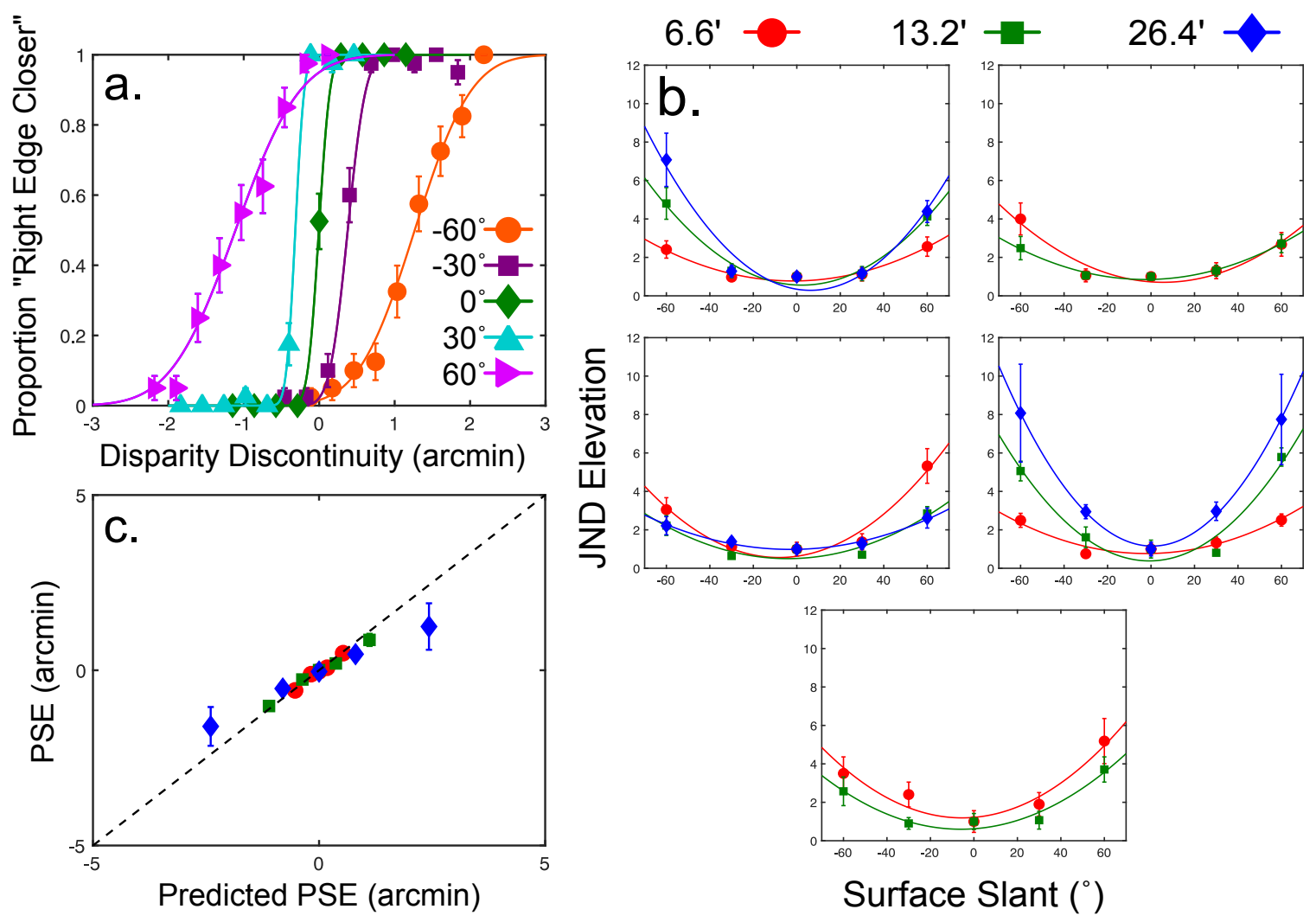

Figure 5. Results of Experiment 2. (a) Example psychometric functions for a single participant, at a gap size of 13.2 arcmin. Error bars show binomial standard errors. (b) Normalised JND ratios as a function of slant for each participant, at each gap size. Error bars show the standard deviation of the bootstrapped distribution of normalised JND estimates. (c) Comparison of predicted with measured PSEs, assuming that responses are based on the judgement of surface relief. Symbols show 
different gap sizes, as in (b). Error bars show standard deviation of the bootstrapped distribution of PSE estimates.

We compared quadratic coefficient values for Experiments 1 and 2 at gap sizes of 6.6 and 13.2 arcmin using a two-way repeated measures ANOVA, using the data for the 5 participants who had completed both experiments. This test showed a significant effect of Experiment $\left(F_{1,4}=8.8432, p=\right.$ $0.041)$, but no significant effect of gap size, or significant interaction $\left(F_{1,4}=0.5698, p=0.4923 ; F_{1,4}\right.$ $=3.8909, p=0.1198$, respectively). One-tailed, related samples t-tests, conducted on the effects of Experiment at each gap size, showed a significant reduction in the quadratic coefficient for Experiment 2 at a gap size of 6.6arcmin $\left(t_{4}=4.496, p=0.0109\right)$, but not at the 13.2 arcmin gap $\left(t_{4}\right.$ $=-0.3644, p=0.7340)$. Three participants also collected data at the 26.4 arcmin gap size for both experiments; a related samples t-test conducted on these data showed no significant difference between experiments $\left(t_{2}=-0.768, p=0.5228\right)$.

Interestingly, while the effect of slant on the precision of discontinuity discrimination was reduced for some conditions in Experiment 2, the use of same-slant stimuli also introduced a new effect. Despite being asked to judge which edge was closer, participants' responses were consistently biased in the direction of the surface slant, with points of subjective equality (PSEs) found at positive (right-near) disparity discontinuity values for negative (right-near) surface slants and vice versa. Similar biases have also recently been reported by Mamassian and Zannoli (2020), for a smaller range of stimulus slants. Such biases are indicative of a response to relative surface structure (i.e. surface relief), rather than egocentric disparity (i.e. disparity relative to the plane of fixation). To evaluate this explanation, we calculated the predicted PSE for each gap size, under the assumption that discontinuity judgements are made on the basis of perceived surface relief. If this assumption were correct, participants' responses would have been based on a comparison of the disparity gradient across the whole surface to the disparity gradient found between neighbouring surface edges. Thus, where the gradient between the edges was less than the surface gradient, the surface slanted behind the plane of fixation was closer. When the gradient between edges was greater than the surface gradient, the surface slanted in front of the fixation plane was closer. Linear fits for the comparison between predicted and measured PSEs for each participant show a consistent relationship, although one that does not fully account for our experimental results. Figure $5 c$ shows this relationship, averaged across participants. Gradients for the linear relationship between predicted and measured PSEs averaged $0.95,0.83$ and 0.59 for gap sizes of $6.6,13.2$ and 26.4 arcmin, respectively. There was, however, substantial variation between participants, with fitted slopes of between 0.66 and 1.3 at the 6.6 arcmin gap size, between 0.36 and 1.09 at the 13.2 arcmin gap, and between 0.32 and 1.06 at the 26.4 arcmin gap.

There are a number of potential explanations for why the shift in PSEs varied between participants. First, the shift in PSEs could be related to perceived, rather than displayed, slant, with some participants substantially under-estimating the slant of the stimulus. This is contrary to earlier findings, however, where perceived slant could not account for relative disparity related effects on stereoacuity (Glennerster \& McKee, 2004). It also fails to account for the overall apparent decrease in biases with increasing gap size. Alternatively, the observed biases may indicate that participants shift between a decision criterion based on surface relief and one based on disparity relative to the fixation plane (cf. Petrov \& Glennerster, 2004, 2006). Such an account also raises the possibility that observed effects of surface slant on the precision of discontinuity discrimination could be, in part, due to this changing decision criterion. To examine this possibility, we conducted a final experiment using stimuli where judgements based on surface relief and egocentric absolute disparity supported the same response. In addition, in section 5, below, we consider whether the observed shift in PSEs may be accounted for by a model that discriminates discontinuity sign based on the encoding of differences in relative disparity.

\section{Experiment 3: Reduced Impairments for Orthogonal Slants}

To better understand the effects of surface slant found in Experiments 1 and 2, Experiment 3 examined the case where the discontinuity was orthogonal to the axis of surface slant. As with the same-slant manipulation in Experiment 2, an absolute disparity account of slant-related impairments 
predicts that surface slant should have the same effect on discontinuity discrimination, regardless of the relative orientation of the slant and discontinuity. In contrast, an account based on relativedisparity selective mechanisms would predict a decrease in slant-related impairments, as in Experiment 2, due to the fact that relative disparity is constant across the stimulus except at the surface discontinuity. In addition, unlike the same-slant stimuli in Experiment 2, relative disparities running orthogonal to the orientation of the discontinuity are not only constant but zero everywhere except at the surface discontinuity; in other words, the change in disparity orthogonal to the discontinuity is equivalent to a fronto-parallel surface. For this stimulus configuration, predicted performance based on surface relief is the same as that based on egocentric disparity, removing any potential effects of changing decision criteria that may have impacted upon performance in Experiment 2. Together these factors suggest that, unless slant-related impairments are due to uncertainty in local absolute disparity measurements, such impairments should be markedly reduced in Experiment 3.

\subsection{Methods}

\subsubsection{Participants}

Experiment 3 was completed by 6 participants, including author RG. All gave written, informed consent as to their participation. All had normal or corrected-to-normal visual acuity, with stereo acuity of at least 1 arcmin, as measured by the RanDot2 stereo test. Five of the 6 participants were experienced psychophysical observers; three had participated in both of the first two studies, and 1 was tested in Experiment 1 only. Non-author participants were naïve as to the purpose of the experiment.

\subsubsection{Stimulus \& Apparatus}

The experimental set up was the same as in all previous experiments. For Experiment 3, however, all stimuli were slanted on a vertical axis $\left( \pm 60^{\circ}, \pm 30^{\circ}\right.$ and $\left.0^{\circ}\right)$. In contrast, surface discontinuities were horizontal such that, depending on the sign of the discontinuity, either the top or the bottom half of the stimulus was closer in depth. Negative disparity values indicate a stimulus where the top half was closer in depth. An example stimulus is shown in Figure 6.

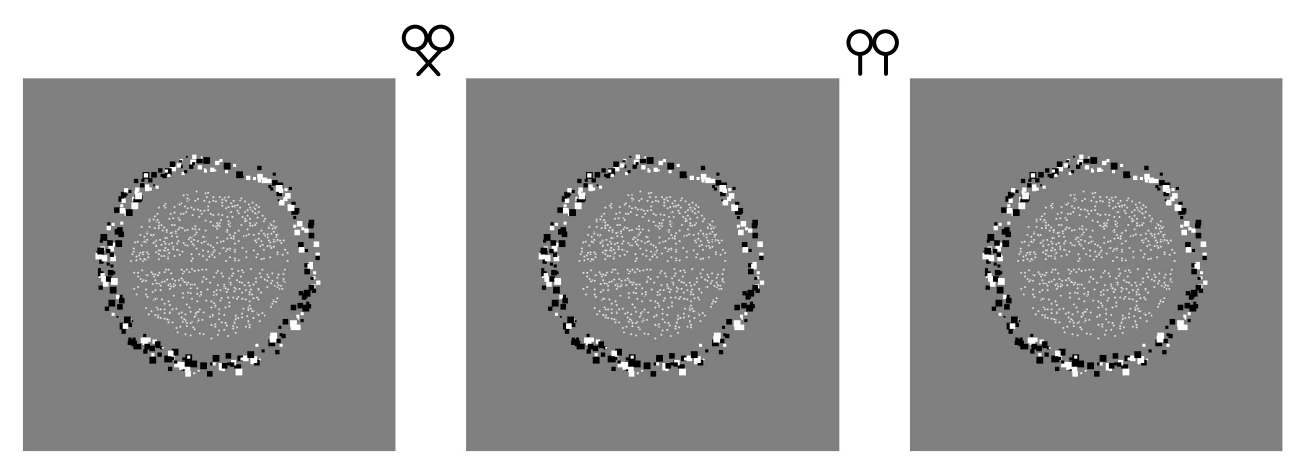

Figure 6. Example of the stimuli used in Experiment 3, arranged for crossed (left \& centre) and uncrossed (centre \& right) fusion. When appropriately fused, the stimulus shows a bottom-near horizontal discontinuity, for a $60^{\circ}$ left-near, right-far slanted surface.

\subsubsection{Design \& Procedure}

The experimental design and procedure were as outlined in Experiments 1 and 2. As before, participants were presented with 9 levels of discontinuity magnitude at differing surface slants. As described above, the orientation of the discontinuity was orthogonal to the surface slant orientation. Discontinuity magnitude was again set for each participant based on the completion of an initial block of practise trials. Values ranged between \pm 2.3 arcmin for $\pm 60^{\circ}$ slants and between \pm 1.14 arcmin for all other slants. Data were collected for combinations of horizontal discontinuities and vertical surface slants only. As before, all stimuli were presented for $300 \mathrm{~ms}$, preceded by the $500 \mathrm{~ms}$ presentation of a fixation cross. Each participant completed a series of blocks, resulting in the collection of a minimum of 20 repeated trials of each experimental stimulus. Each participant collected data for surface gaps of 6.6 arcmin and 13.2 arcmin. 


\section{2 Results \& Discussion}

Cumulative Gaussian distributions were fit to the proportion of "bottom edge closer" responses for each participant and each surface slant. Function slopes were again taken as a measure of JNDs for discontinuity discrimination and were normalised by JNDs for fronto-parallel surfaces (frontoparallel JNDs averaged 8.6 and 4.8 arcsecs across observers, at gap sizes of 6.6 and 13.2 arcmin, respectively, with maximum and minimum values of 84.7 and 3.1 arcsec). Figure 7 plots the normalised JND ratios as a function of surface slant, together with fitted quadratic functions, for each participant, at gap sizes of 6.6 and 13.2 arcmin. Increases in JND ratio were markedly reduced, compared to Experiments 1 and 2, with a mean ratio of $2.27( \pm 0.587)$ for $\pm 60^{\circ}$ slants at a gap size of $6.6 \mathrm{arcmin}$ and a mean ratio of $2.31( \pm 0.343)$ for $\pm 60^{\circ}$ slants at a gap size of 13.2 arcmin. Unlike Experiment 2, there were no consistent shifts in the position of the PSEs.
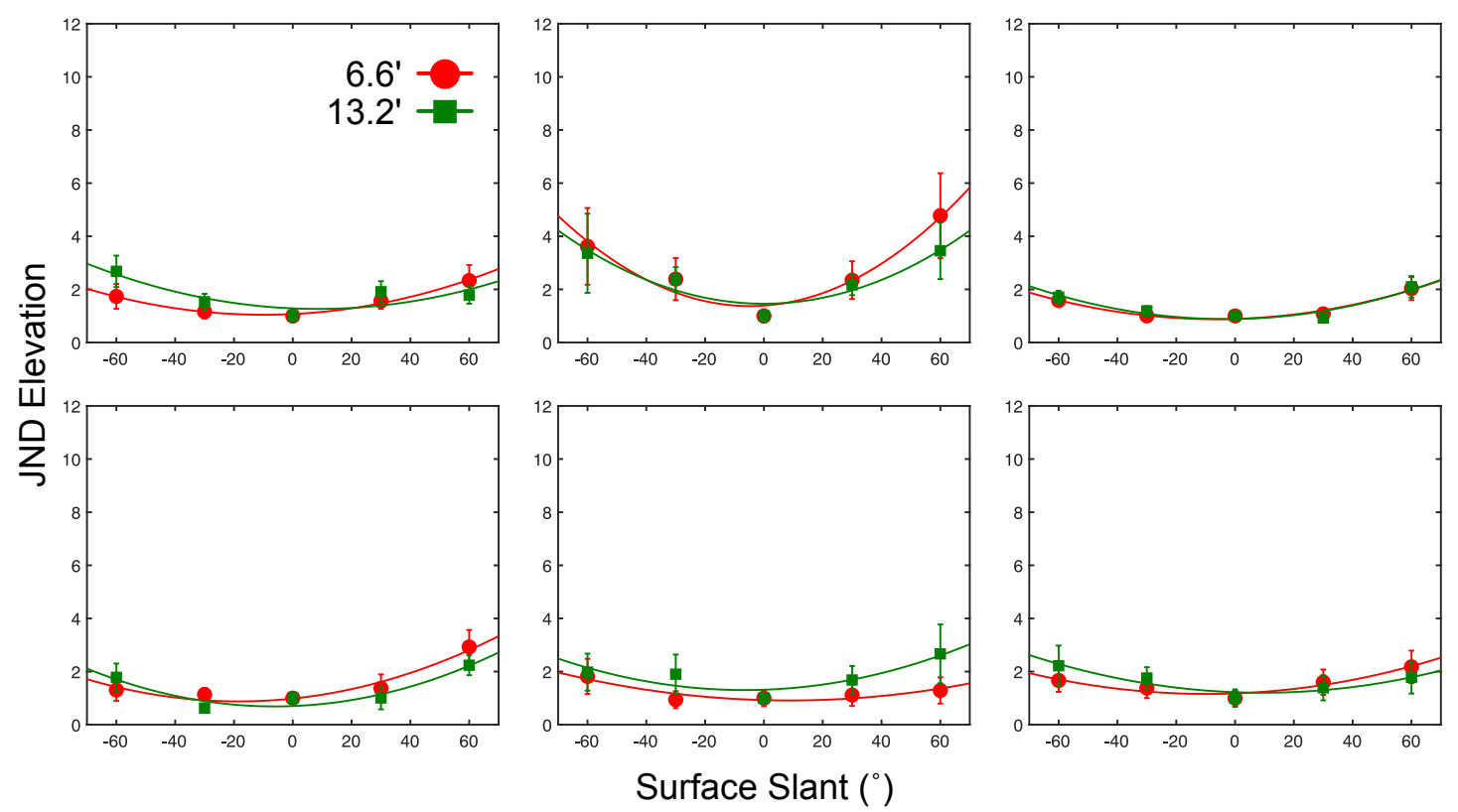

Figure 7. Normalised JND ratios as a function of surface slant in Experiment 3, for each participant and each gap size. Error bars show the standard deviation of the bootstrapped distribution of normalised JND estimates.

Quadratic coefficients describing the effect of slant averaged $0.0003( \pm 0.0002)$ and $0.0003( \pm 0.0001)$ for 6.6 and 13.2 arcmin gaps, respectively. Although the effect of slant on stereoacuity was much reduced, values for the quadratic coefficients were still significantly greater than zero $\left(t_{5}=3.56, p=\right.$ $0.0081 ; t_{5}=6.67, p=0.0005$ for 6.6 and 13.2arcmin gaps, respectively). Adjusted $r^{2}$ values on the quadratic fits ranged between 0.427 and 0.974 across participants and gap sizes, with a mean value of 0.77 . We compared quadratic coefficients found in Experiment 3 with those in Experiment 1, using a two-way repeated measures ANOVA, with Experiment as a between participant variable. There was a significant effect of Experiment $\left(F_{1,10}=47.581, p=0.00004\right)$, but no significant effect of gap size and no significant interaction $\left(F_{1,10}=2.932, p=0.118 ; F_{1,10}=2.783, p=0.126\right.$, respectively). One-tailed, Holm-Bonferroni corrected, two-sample t-tests on the effects of Experiment at each gap size show significant reductions in quadratic coefficients for both 6.6 and 13.2 arcmin gaps $\left(t_{10}=\right.$ $5.764, p=0.00009 ; t_{10}=2.632, p=0.0125$, respectively).

The significant reduction in the effects of slant on discontinuity discrimination show that such effects cannot be explained solely by increased uncertainty in the measurement of local absolute disparities. Instead, the encoding of relative disparities must play a role in driving these slant-related impairments in stereoacuity. The judgement of relative depth at surface discontinuities must, therefore, involve mechanisms measuring changes in relative disparity across space, with surface slant effects arising due to the complexity of these relative disparity changes. 


\section{Modelling Discontinuity Discrimination}

To demonstrate how relative disparity selective mechanisms might account for the patterns of changing stereoacuity found in our experiments, we developed a model for discontinuity discrimination based on the measurement of zero-crossings in second-order differences in disparity (i.e. changes in relative disparity), equivalent in approach to classical models for the detection of luminance-defined edges (Canny, 1986; Ding \& Goshtasby, 2001; Marr \& Hildreth, 1980). This model took as input one-dimensional (1D) representations of the stimuli used in our experiments. Absolute disparities were sampled at 46 random positions for each 1D stimulus, with first and second-order differences in disparity calculated between these positions. Illustrations of these 1D representations are shown in Figure 8a-c, averaged over 1000 stimulus samples, for both frontoparallel surfaces, and for the oppositely slanted surfaces used in Experiment 1. Figure 8d shows second-order disparity differences, orthogonal to the axis of discontinuity, for 1D versions of the stimuli used in Experiments 2 and 3.
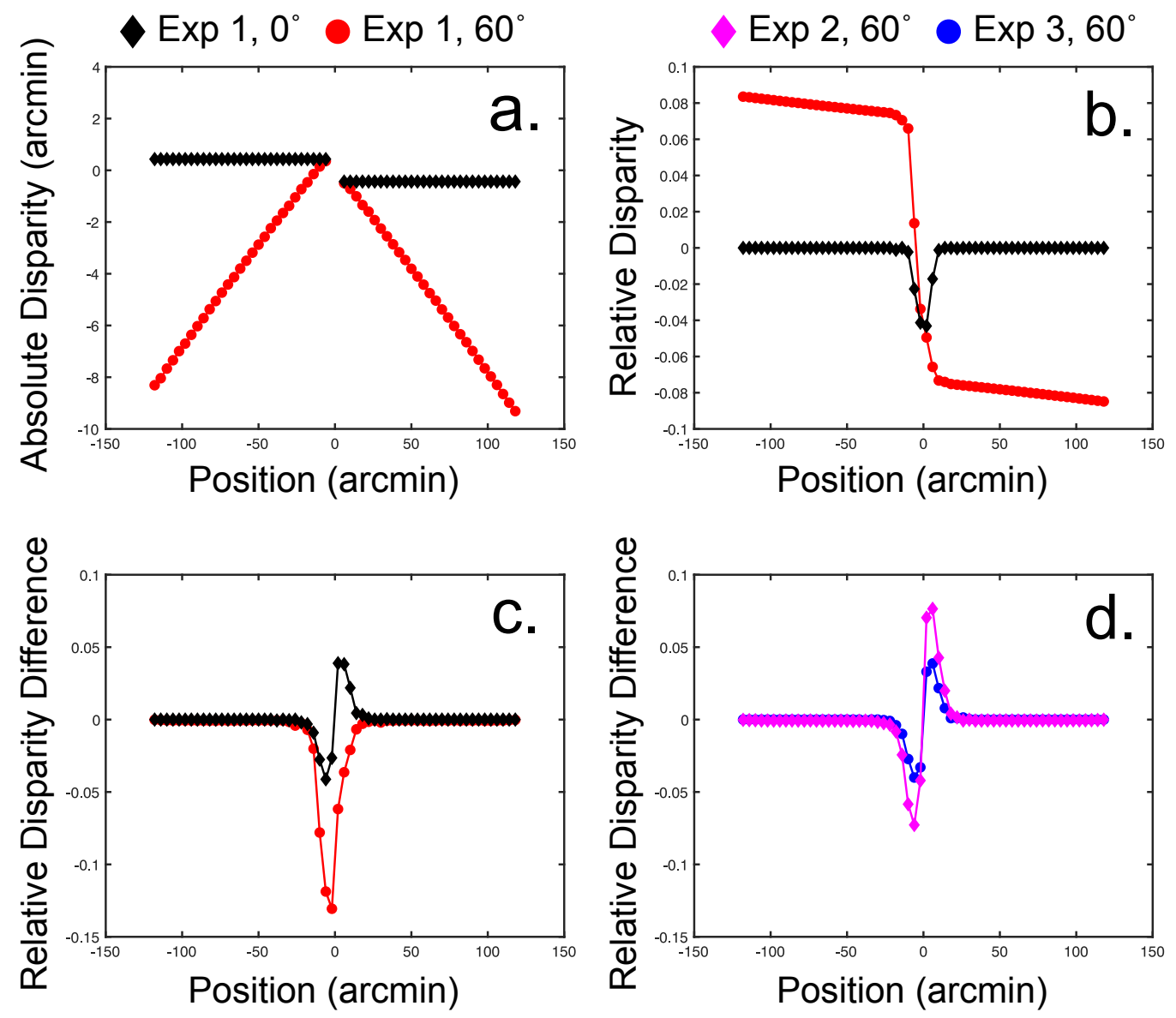

Figure 8. 1D depictions of the stimuli used in Experiments 1-3 as (a) absolute disparities, (b) first order disparity differences (relative disparities) and (c-d) second-order disparity differences. (a) Absolute disparity values for discontinuities with neighbouring fronto-parallel surfaces (black diamonds) and $60^{\circ}$ slanted surfaces in Experiment 1. (b) The same stimuli shown in terms of their relative disparities. (c) Second-order disparity differences for the same stimuli. Note that the frontoparallel surface produces a clear zero-crossing, whereas the $60^{\circ}$ slanted surface does not. (d) Second-order disparity differences for $60^{\circ}$ slanted surfaces in Experiment 2 (magenta diamonds) and Experiment 3 (blue circles). Zero-crossings are visible despite the large surface slants.

The example stimuli provided in Figure 8 show clear differences in the patterns of changing relative disparity between fronto-parallel and slanted surfaces. It is particularly notable that, at equivalent disparity discontinuities, fronto-parallel surfaces gave rise to zero-crossings in second-order differences in disparity, where surfaces with large opposing slants did not. Zero-crossings were also evident for same slant surfaces and surfaces with discontinuities orthogonal to the axis of slant. To 
assess the extent to which these second-order differences might account for discontinuity discrimination performance, we modelled our experiments as the correlation of second-order differences in disparity, with odd-symmetric Gabor filters at multiple spatial scales.

Gabor filters were defined as sinusoidal carriers at spatial frequencies of $0.11,0.23,0.45,0.91$ and 1.81 cycles per degree (equivalent to $0.5,1,2,4$ and 8 cycles per image), enveloped by a Gaussian function with a standard deviation 1.5 times the carrier frequency. Carrier frequencies fall within the boundaries of established functions for disparity frequency sensitivity (typically between 0.05 and 3 cycles per degree), including regions of peak sensitivity at around 0.2 to 0.4 cycles per degree (cf. Banks, et al, 2004; Serrano-Pedraza \& Read, 2010; Tyler, 1975b). Equations for Gabor filters are given in [1]

$G=\mathrm{S} \sin \left(2 \pi \frac{x_{1-n}}{d} \xi\right) e^{\left(-\frac{x_{1-n}^{2}}{2 \sigma^{2}}\right)}$

where $x$ gives the locations of the stimulus dots 1 -n scaled to units of $\pi$ by dividing by the size of the stimulus $d, \xi$ is the spatial frequency of the carrier in cycles per image, $\sigma$ is the standard deviation of the Gaussian envelope and $S$ takes the value \pm 1 in order to modulate the sign of the carrier wave. Carrier frequencies may be converted into units of cycles per degree by dividing the cycles per image value by the $4.4^{\circ}$ angular size of the stimulus. Gabor filters were always centred on zero (i.e. the centre of the disparity discontinuity). Examples of the Gabor filters are shown for a range of spatial scales in Figure 9a.
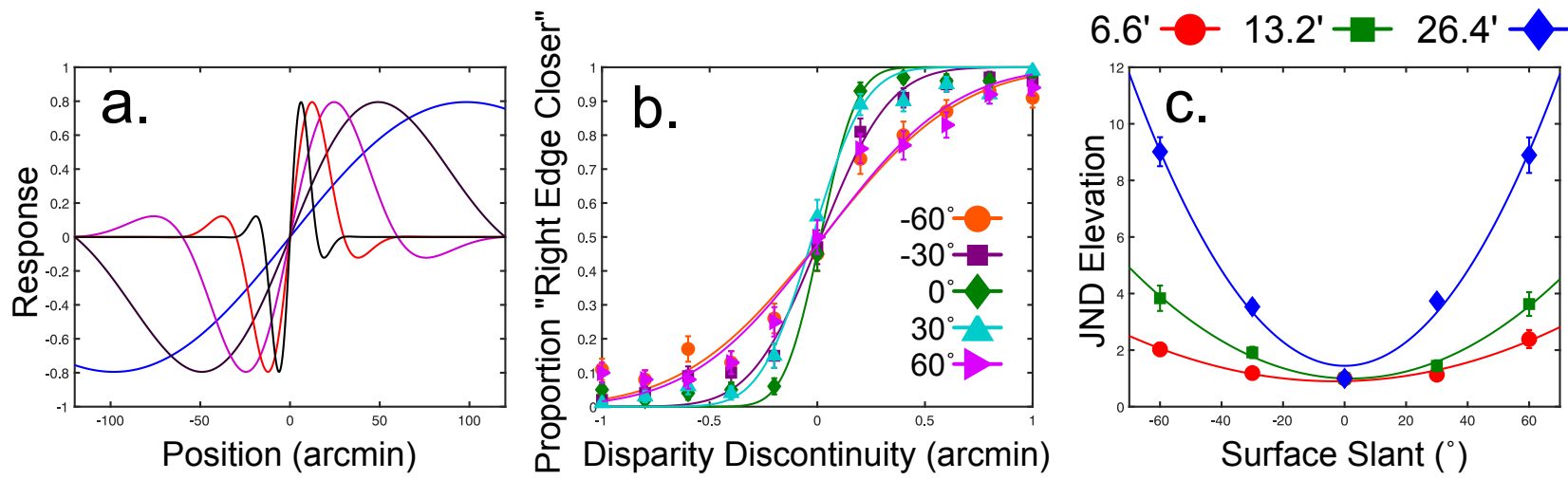

Figure 9. Performance of second-order disparity difference model for Experiment 1. (a) Gabor filters at spatial frequencies of $0.11,0.23,0.45,0.91$ and 1.81 cycles per degree. (b) Psychometric functions showing model performance at each surface slant, for a gap size of 13.2 arcmin. (c) Model performance for all gap sizes and surface slants, shown as normalised JNDs.

To model our experiments, second-order disparity differences were calculated for 1D versions of the stimuli in each experiment, varying edge disparity and surface slant for 1000 repeated trials of each stimulus condition. Second-order stimulus representations were then correlated with the bank of Gabor filters, taking the average correlation across frequency. The sign of filter $S$ resulting in the greater correlation was selected as the sign of the disparity discontinuity. Figure 9b-c show the results of this decision-making process for Experiment 1, as both full psychometric functions for a gap size of 13.2 arcmin and as normalised JNDs across all gap sizes. As is evident from these figures, increases in surface slant increased thresholds for discontinuity discrimination in qualitatively similar manner to human observers. JNDs for fronto-parallel surfaces averaged around 9 arcsec across gap sizes, with normalised JNDs increasing by ratios of between 1.7 and $9.7($ mean $=5.1)$ at slants of $\pm 60^{\circ}$. Notably, however, while the general pattern and scale of slant-related JND elevations were well matched with human observers, our model predicted a clear pattern of increasing slantrelated impairment with increasing gap size. We found no consistent evidence of this in our observers. In our model, this effect is likely due to an increasing reliance on lower frequency filters as gap size increased. While such processes may underlie similar effects in some of our observers, others are clearly able to make use of more reliable stimulus information. Reduced effects of surface 
slant at large gap sizes for these observers may reflect alternative processing strategies, for example through a comparison of the surface edge with the fronto-parallel fixation plane.

Model performance for Experiments 2 and 3 is shown in Figure 10, as example psychometric functions, normalised JNDs and, for Experiment 2, as a comparison of predicted with measured PSEs. In both Experiments 2 and 3, our zero-crossing model predicts an absence of slant-related impairments in discontinuity discrimination (Figure 10a-b, d-e). Thresholds for fronto-parallel stimuli again averaged around 9 arcsec. While reduced slant effects were found in both of these experiments, such effects were not entirely absent. As such, processes based on the detection of second-order differences in disparity cannot wholly account for these slant effects; lower-level increases in the uncertainty of absolute and/or relative disparity measurements would instead seem to play at least a small part in these slant-related impairments. Interestingly, our model also does well in predicting the observed pattern of slant-related changes in PSE in Experiment 2, including increases in the change in PSE with increasing gap size (see Figure 10c). Gradients for linear fits of the relationship between 'surface relief' predicted PSEs and modelled PSEs were 1.026 (with 95\% Cls ranging from 0.9457 to 1.107$), 1.006$ (95\% Cl of $0.9605-1.052)$ and 0.9562 (95\% Cls of $0.9347-$ 0.9777 ) at gap sizes of $6.6,13.2$ and 26.4 arcmin, respectively. Thus, although our zero-crossing model produced a shift in PSEs, this was somewhat larger than that found in our psychophysical data. As with gap size effects, this may reflect participants' adoption of alternative strategies for judging discontinuity direction. We consider these issues in greater detail, below. Despite these failures to account for individual differences in both gap size effects and PSE shifts, the ability of our model to account for general changes in the pattern of slant-related biases and threshold elevations across all three experiments suggest that these effects are largely attributable to mechanisms selective for differences in relative disparity.
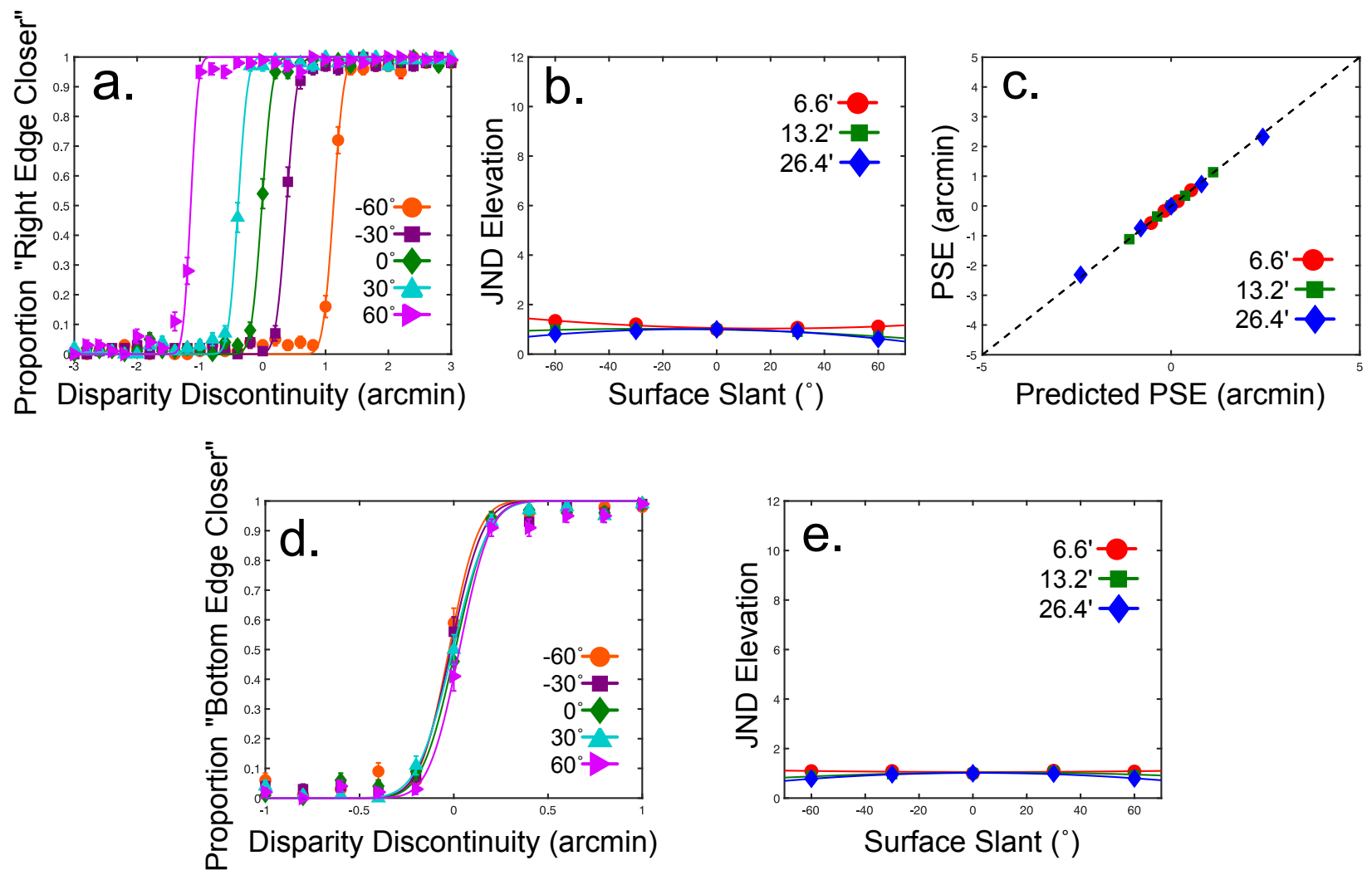

Figure 10. Performance of the second-order disparity difference model in Experiments 2 and 3. (a) Model performance for Experiment 2, shown as psychometric functions for a gap size of 13.2 arcmin. (b) Normalised JNDs as a function of surface slant for each gap size. (c) Model PSEs for each gap size, plotted against predicted PSEs under the assumption that discontinuities are discriminated based on judgements of surface relief. (d) Model performance for Experiment 3, shown as psychometric functions for a gap size of 13.2 arcmin. (e) Normalised JNDs as a function of surface slatn for each gap size. 


\section{General Discussion}

The results reported here show that, just as intervening surface structures can impair stereoacuity judgements, so too can adjacent surface structures limit the ability to discriminate the sign of a depth discontinuity between neighbouring edges. Slanted surfaces on either side of a discontinuity impair stereoacuity judgements. The scale of these effects is dependent upon the specific arrangement of stimulus surfaces in a manner consistent with the effects of mechanisms for the encoding of relative disparity. Below, we consider the interpretation of these stereoacuity impairments in relation to earlier results and, in light of our modelling results, consider the nature of the relative disparity selective mechanisms that drive them.

\subsection{Limitations in Discontinuity Discrimination}

The finding that adjacent surface slants impair disparity discontinuity discrimination is consistent with multiple previous results showing that performance in psychophysical tasks is affected by different disparity-defined surface structures. For intervening surface structures, multiple researchers have shown that slanted surfaces lead to an underestimation of depth (Cammack \& Harris, 2016; Deas \& Wilcox, 2014, 2015; Goutcher et al, 2018; McKee, 1983; Mitchison \& Westheimer, 1984; Westheimer, 1979), while the arrangement of adjacent surface structures has been shown to affect the perception of disparity-defined slant (Wardle \& Gillam, 2016). Goutcher and colleagues (2018) argued that the encoding of relative disparities plays a critical role in driving these effects, while Deas and Wilcox $(2014,2015)$ and Mamassian and Zannoli (2020) have also provided evidence of the importance of gestalt grouping processes. The results reported here, together with the behaviour of our zero-crossing model, provide further support for the importance of these relative disparity processes in driving stereoacuity performance. Slant-related threshold elevations were dependent on specific surface configurations in a manner consistent with the operation of mechanisms selective for changes in relative disparity.

In contrast, we find limited evidence that surface-related effects on stereoacuity depend critically upon uncertainty in the early measurement of absolute disparities. Early measurement mechanisms of this kind have been suggested to account for limitations in disparity frequency sensitivity (Banks et al, 2004), disparity gradient limits (Filippini \& Banks, 2009) and amplitude limits on the detection of disparity-defined corrugations (Allenmark \& Read, 2010, 2011). For surface-related effects, disparity averaging processes, operating at the early measurement level, have been proposed as an explanation for the underestimation of depth in stimuli with continuous, smooth changes in disparity (Cammack \& Harris, 2016). Our results showed, however, that slant-related elevations in discontinuity discrimination thresholds depended critically on the arrangement of surfaces around the discontinuous edge, rather than on surface slant itself.

That is not to suggest, however, that the uncertainty of absolute disparity measurements, or indeed relative disparity measurements, plays no role in these slant-related effects. Threshold elevation was not completely eliminated in Experiments 2 or 3, suggesting at least some role for slant-dependent measurement noise in determining the sign of the disparity discontinuity. This is further supported by the fact that this residual elevation was not evident in the results of our zero-crossing model. An adapted version of our model could account for these remaining effects through the introduction of slant-dependent additive noise on the disparities of stimulus dots. Importantly, such noise would provide a common additive effect on discrimination thresholds across all experiments and could not, in isolation, account for the increased threshold elevations found in Experiment 1. Slant dependent noise of this kind could arise due to the effects of shearing and/or scaling disparities on the crosscorrelation-like measurement of absolute disparities in V1 (Filippini \& Banks, 2009), or due to the tuning properties of relative disparity selective neurons in V2 and/or V4 (Fang et al, 2018; Hinkle \& Conner, 2002; Thomas et al, 2002; Umeda et al, 2007).

Finally, our results provide no consistent evidence to support the role of surface grouping processes in discontinuity discrimination. At least for our stimuli. In Experiment 1, if slant-related effects were due to surface grouping processes operating across the change in sign of slant, threshold elevation should have decreased as the gap between surfaces increased. Although some participants showed effects of this kind, it was not a consistent pattern. Instead, some showed more threshold elevation 
as the gap between the surfaces increased, consistent with the behaviour of our zero-crossing model. The lack of consistent gap size effects may suggest individual differences in the scales at which observers encode relative disparity differences, or that some observers are able to make increased use of surrounding information used to define the fixation plane (see Figures 1, 4 and 6). Further empirical study is required to understand the impact of proximity on the discrimination of disparity discontinuities.

\subsection{Mechanisms for the Encoding of Relative Disparity}

To account for patterns of slant-dependent elevation of discontinuity discrimination thresholds, we modelled task performance through the measurement of local changes in relative disparity. These local relative disparity changes were detected via odd-symmetric Gabor filters. Tyler (1975a) referred to disparity frequency tuned filters of this kind as the 'hypercyclopean' stage of processing, with subsequent findings (Serrano-Pedraza \& Read, 2010; Tyler \& Kontsevich, 2001) showing the importance of such processing in accounting for the effects of orientation in disparity processing. Our results suggest that hypercyclopean mechanisms of this kind play a critical role in determining the patterns of slant-dependent threshold elevations observed in our experiments.

The use of mechanisms tuned to differences in relative disparity would provide the visual system with the means to encode complex surface structures, for example patterns defined by depth relief, and make comparisons between structures, regardless of large degrees of variability in viewing angle. Sensitivity for such depth relief patterns is consistent with the results shown in Experiment 2. More broadly, however, the use of such mechanisms is consistent with the idea that, even for the encoding of disparity discontinuities, the visual system makes the assumption that disparity changes are piecewise smooth (cf. Goutcher \& Hibbard, 2010; Goutcher \& Mamassian, 2005; Marr \& Poggio, 1976, 1979; McKee \& Mitchison, 1988). Thus, depth discontinuities are not defined by, for example, image areas containing large disparity gradients per se, but by image areas where a constant disparity gradient is punctuated by a large change. Mechanisms tuned to changes in relative disparity, such as the zero-crossing model applied here, are well-suited to the detection of exactly these structures.

The proposal that variations in surface structure, such as depth relief, might be encoded by neural mechanisms selective for changes in relative disparity raises questions as to the spatial scale at which such surface structures are processed. Here, we have considered mechanisms within a range of 0.12 to 1.81 cycles per degree, motivated by earlier findings on the detection of cyclopean gratings (cf. Serrano-Pedraza \& Read, 2010). One would expect such mechanisms to depend upon responses from neural populations earlier in the visual hierarchy, such as relative disparity selective neurons in V2 (Bredfeldt \& Cumming, 2006; Thomas et al, 2002) and, ultimately, neurons in V1 selective for absolute disparity (Cumming \& Parker, 1999; DeAngelis et al, 1991; Ohzawa et al, 1990). Later visual areas, such as V4 and other ventral areas such as IT, TEO and TE are likely involved in the specific encoding of disparity discontinuities and the use of disparity-derived signals for image segmentation (Fang et al, 2018; Roe et al, 2012; Uka, Tanaka, Yoshiyama, Kato \& Fujita, 2000; Umeda et al, 2007; Verhoef, Bohon \& Conway, 2015).

At the level of $\mathrm{V} 1$ disparity selective neurons, multiple authors have proposed correlations between receptive field size and absolute disparity selectivity (Allenmark \& Read, 2011; Prince \& Eagle, 1999; Smallman \& MacLeod, 1994), with receptive field size having also been proposed as a limiting factor on disparity frequency sensitivity (Banks et al, 2004; Nienborg, Bridge, Parker \& Cumming, 2004). This suggests that the proposed hypercyclopean mechanisms should be fed by different sizes of absolute disparity detectors, depending on the disparity frequency to which they are tuned. Future research should examine how these factors of absolute disparity and disparity frequency combine to define the selectivity of proposed hypercyclopean processes.

An alternative possibility to these zero-crossing dependent processes can be found in the work of Glennerster and colleagues (Glennerster \& McKee, 1999, 2004; Petrov \& Glennerster, 2004, 2006). These authors have argued for mechanisms that compare the disparity of isolated points to a measured, or inferred, reference plane (see also Hornsey et al, 2016). Such mechanisms could help to account for some of the slant-dependent effects found in Experiment 2 if the reference surface 
used to judge discontinuity direction varied on a trial-by-trial basis between the stimulus slant and the fronto-parallel fixation plane. Under this account, however, the detection of zero-crossings would still offer a robust means of encoding disparity discontinuities and surface relief structure, although they would be measured as zero-crossings relative to the reference plane. One way in which our model could implement such processes is by filling in the gap between surface edges with an estimate of the reference plane structure. Such 'filled in' areas would likely require such a model to consider the uncertainty with which the reference plane was encoded and would necessitate a more complex decision rule that measured the change in sequences of zero-crossing over space. A similar mechanism was proposed by Goutcher and colleagues (Goutcher et al, 2018) to account for discontinuity-related effects of quantitative perceptions of suprathreshold changes in disparity.

\subsection{On the Ecological Validity of Opposing Slant Discontinuities}

One critical issue arising from our findings is the extent to which slant-related impairments in discontinuity discrimination affect the processing of disparity in real world scenes. Given that we expect that our visual system is well-tuned to the statistical structure of our environment (cf. Burge \& Geisler, 2014; Chauhan, Héjja-Brichard \& Cottereau, 2020), one would hope that the kinds of opposing slant discontinuities employed in this paper are relatively rare in the real world. Specifically, while opposing slants are likely to be common in many scenes (e.g. at object corners), it seems unlikely that these locations would co-occur with parallel discontinuities with any regularity. Indeed, these stimuli appear to violate a fundamental assumption that forms an explicit or implicit part of multiple accounts of disparity processing, that surfaces are piecewise smooth (Marr \& Poggio, 1976, 1979). The efficacy of this assumption is immediately apparent when we consider the suitability of zero-crossing-based measurements in the judgement of surface relief, as seen in Experiment 2, where the detection of discontinuities can be made against otherwise continuous surfaces. For more complex arrangements, such as the detection of disparity discontinuities at object boundaries, although the surfaces themselves may be differently slanted close to the boundary edge, these slants are likely to be small relative to the disparity between objects.

If zero-crossings are indeed an efficient way to encode disparity discontinuities in natural scenes, stimuli that impair these mechanisms, such as those found in Experiment 1, could help to point to effective forms of depth-based camouflage. Cammack and Harris (2016) have previously suggested that smooth edges between objects and their background could offer a form of depth camouflage through both reduced estimates of relative depth and the reduced visibility of depth-edges. Similarly, animals situated close to depth inflection points could exploit the kind of slant-related stereoacuity impairments found in our experiments. This form of camouflage would allow depth differences between the animal and its background to be hidden by more complex patterns of variation in depth.

\subsection{Conclusions}

This paper has presented the results of a series of psychophysical experiments, which show that disparity discontinuity discrimination thresholds are affected by the slant of adjacent surface structures. These effects are consistent with a mechanism that determines the sign of a disparity discontinuity through the measurement of local changes in relative disparity. Slant-dependent discontinuity discrimination effects thus appear to be primarily driven by these relative disparity related processes rather than by early noise in the measurement of absolute disparities.

\section{References}

Allenmark, F., \& Read, J.C.A. (2010). Detectability of sine-versus square-wave disparity gratings: A challenge for current models of depth perception. Journal of Vision, 10(8):17, 1-16.

Allenmark, F., \& Read, J.C.A. (2011). Spatial stereoresolution for depth corrugations may be set in primary visual cortex. PLoS Computational Biology, 7(8): e1002142.

Badcock, D.R. \& Schor, C.M. (1985). Depth-increment detection function for individual spatial channels. Journal of the Optical Society of America, A, 2, 1211-1215.

Banks, M.S., Gepshtein, S., \& Landy, M.S. (2004). Why is spatial stereoresolution so low? Journal of Neuroscience, 24(9), 2077-2089.

Blakemore, C. (1970). The range and scope of binocular depth discrimination in man. Journal of Physiology, 211, 599-622.

Brainard, D.H. (1997). The psychophysics toolbox. Spatial Vision, 10, 433-436. 
Bredfeldt, C.E. \& Cumming, B.G. (2006). A Simple Account of Cyclopean Edge Responses in Macaque V2. Journal of Neuroscience. 26(29), 7581-7596.

Burge, J. \& Geisler, W.S. (2014). Optimal disparity estimation in natural stereo images. Journal of Vision, 14(2):1, 1-18, doi:10.1167/14.2.1

Canny, J. (1986). A computational approach to edge detection. IEEE Transactions on Pattern Analysis and Machine Intelligence, 8(6), 679-698.

Cammack, P., \& Harris, J.M. (2016). Depth perception in disparity-defined objects: finding the balance between averaging and segregation. Philosophical Transactions of the Royal Society of London, Series B, 371(1697), 20150258.

Chauhan, T., Héjja-Brichard, Y. \& Cottereau, B.R. (2020). Modelling binocular disparity processing from statistics in natural scenes. Vision Research, 176, 27-39.

Coutant, B.E. \& Westheimer, G. (1993). Population distribution of stereoscopic ability. Ophthalmic and Physiological Optics, 13, 3-7.

Cumming, B.G. \& Parker, A.J. (1999). Binocular neurons in V1 of awake monkeys are selective for absolute, not relative, disparity. Journal of Neuroscience, 19, 5602-5618.

DeAngelis, G.C., Ohzawa, I., \& Freeman, R.D. (1991). Depth is encoded in the visual cortex by a specialized receptive field structure. Nature, 352(6331), 156-159.

Deas, L.M., \& Wilcox, L.M. (2014). Gestalt grouping via closure degrades suprathreshold depth percepts. Journal of Vision, 14(9):14, 1-13.

Deas, L.M., \& Wilcox, L.M. (2015). Perceptual grouping via binocular disparity: the impact of stereoscopic good continuation. Journal of Vision, 15(11):11, 1-13.

Ding, L \& Goshtasby, A. (2001). On the Canny edge detector. Pattern Recognition. 34, 721-725.

Fang, Y., Chen, M., Xu, H., Li, P., Han, C., Hu, J., Zhu, S., Ma, H. \& Lu, H.D. (2018). An Orientation Map for Disparity-Defined Edges in Area V4. Cerebral Cortex. 29(2), 666-679

Field, D.J. \& Hayes, A. (2004). Contour Integration and the Lateral Connections of V1 Neurons in L.M. Chalupa \& J.S. Werner (Eds.) The Visual Neurosciences (pp. 1069-1079). Cambridge, MA: MIT Press.

Field, D.J., Hayes, A. \& Hess, R.F. (1993). Contour integration by the human visual system: Evidence for a local "association field". Vision Research, 33, 173-193.

Filippini, H.R., \& Banks, M.S. (2009). Limits of stereopsis explained by local crosscorrelation. Journal of Vision, 9(1):8, 1-18.

Fleet, D.J., Wagner, H., \& Heeger, D.J. (1996). Neural encoding of binocular disparity: energy models, position shifts and phase shifts. Vision Research, 36(12), 1839-1857.

Georgeson, M.A., Freeman, T.C.A. \& Scott-Samuel, N.E. (1996). Sub-pixel accuracy: Psychophysical validation of an algorithm for fine positioning and movement of dots on visual displays. Vision Research, 36(4), 605-612.

Glennerster, A. \& McKee, S.P. (1999). Bias and sensitivity of stereo judgements in the presence of a slanted reference plane. Vision Research. 39, 3057-3069.

Glennerster, A. \& McKee, S.P. (2004). Sensitivity to depth relief on slanted surfaces. Journal of Vision, 4, 378-387.

Goutcher, R. \& Hibbard, P.B. (2010). Evidence for relative disparity matching in the perception of an ambiguous stereogram. Journal of Vision. 10(12):35, 1-16.

Goutcher, R., \& Hibbard, P.B. (2014). Mechanisms for similarity matching in disparity measurement. Frontiers in Psychology, 4, 1014, 1-11.

Goutcher, R. \& Hibbard, P.B. (2020). Impairment of Cyclopean Surface Processing by DisparityDefined Masking Stimuli. Journal of Vision,

Goutcher, R., Connolly, E. \& Hibbard, P.B. (2018). Surface continuity and discontinuity bias the perception of stereoscopic depth. Journal of Vision, 18(12):13, 1-15. doi:10.1167/18.12.13

Goutcher, R. \& Mamassian, P. (2005). Selective biasing of stereo correspondence matching in an ambiguous stereogram. Vision Research, 45, 469-483.

Hinkle, D.A. \& Conner, C.E. (2002). Three-dimensional orientation tuning in macaque area V4. Nature Neuroscience, 5, 665-670.

Hornsey, R.L., Hibbard, P.B. \& Scarfe, P. (2016). Binocular Depth Judgments on Smoothly Curved Surfaces. PLoS One, 11(11): e0165932.

Howard, H.J. (1919). A test for the judgment of distance. American Journal of Ophthalmology, 2, 656-675.

Kleiner, M., Brainard, D., \& Pelli, D. (2007). What's new in Psychtoolbox-3. Perception, 36S, 14. 
McKee, S.P. (1983). The spatial requirements for fine stereoacuity. Vision Research, 23(2), 191198.

McKee, S.P. \& Mitchison, G.J. (1988). The role of retinal correspondence in stereoscopic matching. Vision Research, 28(9), 1001-1012.

Mamassian, P. \& Zannoli, M. (2020). Sensory loss due to object formation. Vision Research, 174, 22-40.

Marr, D. \& Hildreth, E. (1980). Theory of edge detection. Proceedings of the Royal Society of London, Series B: Biological Sciences. 207, 187-217.

Marr, D. \& Poggio, T. (1976). Cooperative computation of stereo disparity. Science, 194, 283-287.

Marr, D. \& Poggio, T. (1979). A computational theory of human stereo vision. Proceedings of the Royal Society of London, Series B: Biological Sciences, 204, 301-328.

Mitchison, G.J., \& Westheimer, G. (1984). The perception of depth in simple figures. Vision Research, 24(9), 1063-1073.

Nienborg, H., Bridge, H., Parker, A.J. \& Cumming, B.G. (2004). Receptive Field Size in V1 Neurons Limits Acuity for Perceiving Disparity Modulation. Journal of Neuroscience, 24(9), 2065-2076.

Ohzawa, I., DeAngelis, G.C., \& Freeman, R.D. (1990). Stereoscopic depth discrimination in the visual cortex: neurons ideally suited as disparity detectors. Science, 249(4972), 1037-1041.

Pelli, D.G. (1997). The VideoToolbox software for visual psychophysics: Transforming numbers into movies. Spatial Vision, 10(4), 437-442.

Petrov, Y., \& Glennerster, A. (2004). The role of a local reference in stereoscopic detection of depth relief. Vision Research, 44(4), 367-376.

Petrov, Y., \& Glennerster, A. (2006). Disparity with respect to a local reference plane as a dominant cue for stereoscopic depth relief. Vision Research, 46(26), 4321-4332.

Prince, S.J.D. \& Eagle, R.A. (1999). Size-disparity correlation in human binocular depth perception. Proceedings of the Royal Society of London, Series B: Biological Sciences. 266(1426), 1361-1365.

Qiu, F.T. \& von der Heydt, R. (2005). Figure and Ground in the Visual Cortex: V2 Combines Stereoscopic Cues with Gestalt Rules. Neuron, 47, 155-166.

Read, J.C.A. (2005). Early computational processing in binocular vision and depth perception. Progress in Biophysics and Molecular Biology, 87, 77-108.

Roe, A.W., Chelazzi, L., Connor, C.E., Conway, B.R., Fujita, I., Gallant, J.L., Lu, H. \& Vanduffel, W. (2012). Towards a Unified Theory of Visual Area V4. Neuron, 74, 12-29.

Samonds, J.M., Potetz, B.R. \& Lee, T.S. (2009). Cooperative and Competitive Interactions Facilitate Stereo Computations in Macaque Primary Visual Cortex. Journal of Neuroscience, 29(50), 15780-15795.

Samonds, J.M., Tyler, C.W. \& Lee, T.S. (2016). Evidence of Stereoscopic Surface Disambiguation in the Responses of V1 Neurons. Cerebral Cortex, 27, 2260-2275.

Serrano-Pedraza, I. \& Read, J.C.A. (2010). Multiple channels for horizontal, but only one for vertical corrugations? A new look at the stereo anisotropy. Journal of Vision, 10(12):10, 1-11.

Smallman, H.P. \& MacLeod, D.I. (1994). Size-disparity correlation in stereopsis at contrast threshold. Journal of the Optical Society of America, A. 11(8), 2169-2183.

Thomas, O.M., Cumming, B.G. \& Parker, A.J. (2002). A specialization for relative disparity in V2. Nature Neuroscience. 5(5), 472-478.

Tyler, C.W. (1975a). Stereoscopic tilt and size aftereffects. Perception, 4, 187-192.

Tyler, C.W. (1975b). Spatial Organization of Binocular Disparity Sensitivity. Vision Research, 15, 583-590.

Tyler, C.W. \& Kontsevich, L.L. (2001). Stereoprocessing of cyclopean depth images: horizontally elongated summation fields. Vision Research, 41, 2235-2243.

Uka, T., Tanaka, H., Yoshiyama, K., Kato, M. \& Fujita, I. (2000). Disparity selectivity of neurons in monkey inferior temporal cortex. Journal of Neurophysiology, 84, 120-132.

Umeda, K., Tanabe, S. \& Fujita, I. (2007). Representation of Stereoscopic Depth Based on Relative Disparity in Macaque Area V4. Journal of Neurophysiology. 98, 241-252.

Verhoef, B-E., Bohon, K.S. \& Conway, B.R. (2015). Functional architecture for disparity in macaque inferior temporal cortex and its relationship to the architecture for faces, color, scenes and visual field. Journal of Neuroscience, 35, 6952-6968. 
Verhoef, B-E., Vogels, R. \& Janssen, P. (2016). Binocular depth processing in the ventral visual pathway. Philosophical Transactions of the Royal Society, Series B. 371, 20150259.

Wardle, S.G. \& Gillam, B.J. (2016). Gradients of relative disparity underlie the perceived slant of stereoscopic surfaces. Journal of Vision, 16(5):16, 1-13.

Westheimer, G. \& McKee, S.P. (1978). Stereoscopic acuity for moving retinal images. Journal of the Optical Society of America, 68, 450-455.

Westheimer, G. (1979). The spatial sense of the eye. Proctor Lecture. Investigative Ophthalmology and Visual Science, 18, 893-912.

\section{Acknowledgements}

This research was supported in part by a Carnegie Trust travel grant awarded to RG. 